\title{
REPRESENTACIONALISMO, ATENCIÓN Y PROPIEDADES APARENTES
}

\author{
Francisco Pereira Gandarillas ${ }^{1}$ \\ Universidad Alberto Hurtado \\ fpereira@uahurtado.cl
}

\begin{abstract}
RESUMEN / ABSTRACT
Uno de los principales desafíos que debe enfrentar el representacionalismo es dar cuenta de manera consistente de la posibilidad de escenarios en que la atención encubierta altera la fenomenología de nuestras experiencias visuales. Este trabajo intenta poner de relieve los compromisos y dificultades internas de tres estrategias representacionales diferentes para enfrentar este desafío: la estrategia de contenidos indeterminados, la estrategia de la ilusión masiva y el proyecto narcisista que apela a propiedades relacionales indexadas a la atención. Se argumentará que este último posee virtudes comparativas evidentes que la literatura ha ignorado y que justifican su desarrollo en profundidad.
\end{abstract}

PALABRAS ClaVE: representacionalismo, atención, fenomenología, contenido, percepción.

\section{REPRESENTATIONALISM, ATTENTION AND APPARENT PROPERTIES}

One of the main challenges that representationalism must face is to consistently account for the possibility of scenarios in which covert attention alters the phenomenology of our visual experiences. The present work tries to highlight the internal commitments and difficulties of three different representational strategies to face this challenge: the strategy of indeterminate content, the strategy of mass illusion and the narcissistic project that appeals to relational properties indexed to attention. It will be argued that the latter has evident comparative virtues that the literature has ignored and that justify its in-depth development.

KEYWORDS: representationalism, attention, phenomenology, content, perception.

\section{El desafio atencional}

RA El representacionalismo puede ser caracterizado mínimamente como una tesis filosófica según la cual el carácter fenoménico de una experiencia-es decir, el cómo es para un organismo tener dicha experiencia- superviene y está determinado totalmente por el contenido representacional de la misma. La relación de superveniencia garantiza que si dos experiencias difieren en su carácter fenoménico, entonces difieren

Agradezco el apoyo financiero otorgado por el Proyecto Fondecyt Regular 1200197, ANID, Gobierno de Chile. 
en su contenido representacional. Asimismo, hablar de "determinación total" implica aceptar una caracterización pura según la cual es el contenido representacional de una experiencia el que por sí mismo explica y da cuenta exhaustivamente de cómo es para un organismo tener dicha experiencia ${ }^{2}$. En lo que sigue me referiré exclusivamente a esta caracterización pura del representacionalismo, ya que es la que domina las discusiones contemporáneas (Dretske 1995, Lycan 1996, Tye 1995) y restringiré mis comentarios al ámbito específico de la modalidad experiencial visual.

Al evaluar las implicancias del representacionalismo podemos fácilmente vislumbrar potenciales problemas. Uno de ellos es la eventual existencia de experiencias que instancien propiedades fenoménicas diferentes, pero que posean exactamente las mismas propiedades representacionales. Acotar la discusión a una clase de estados mentales y a una modalidad específica como la visual es de suma importancia en este contexto. Uno puede estipular sin dificultad diferencias fenomenológicas entre estados mentales de una clase diferente. Por ejemplo, hay una diferencia fenomenológica entre ver que hay una mancha azul en la muralla blanca y creer que hay una mancha azul en la muralla blanca. Pese a la identidad del contenido proposicional especificado a continuación de la clausula "que" - hay una mancha azul en la muralla blanca- la fenomenología de estados mentales como la creencia y la percepción visual no son equivalentes. Lo mismo parece ser válido para estados o eventos de una misma clase genérica, como podría ser una experiencia sensorial, si lo que está en juego son modalidades diversas. Por ejemplo, hay diferencia fenomenológica existente entre ver que María sube las escaleras y escuchar que María sube las escaleras. El contenido representacional es el mismo, pero la diferencia fenomenológica probablemente reside en la diferencia de modalidad.

El desafío que nos interesa discutir en este artículo es uno que impone una diferencia fenomenológica bajo una modalidad sensorial específica en que supuestamente el contenido representacional es el mismo. ¿Cuándo podría ocurrir esto? ¿Es esto una mera posibilidad teórica o disponemos de antecedentes empíricos? Chalmers fue uno de los primeros filósofos en plantear no solo este escenario en términos hipotéticos, sino en sugerir un ejemplo fundado en el fenómeno del cambio atencional:

...los casos potenciales más plausibles de experiencias visuales fenomenológicamente diferentes con el mismo contenido representacional involucran diferencias en la atención. Cambios en la atención claramente hacen una diferencia fenoménica a las experiencias visuales... Por ejemplo, uno podría mirar dos puntos lumínicos

2 Esta caracterización genérica de lo que es el carácter fenoménico logra captar lo que Nagel (1974) alguna vez estipuló como el what-it-is-likeness propio de un evento o estado mental. Chalmers, por ejemplo, afirma que "el carácter fenoménico de una experiencia perceptual es el cómo es tener esa experiencia" (2006, p. 50) y Tye al dar cuenta de cómo es tener experiencias diversas como sentir hambre, ver un azul púrpura o sentir picazón en un brazo, no duda en afirmar que "en tanto cómo es tener cada una de estas experiencias es diferente, las experiencias difieren en carácter fenoménico" (2009, p. 2). 
rojos contra un fondo negro y cambiar la atención de uno a otro. Aquí no es obvio que exista una diferencia representacional entre los casos (Chalmers 2010, pp. 348-349).

Chalmers sostiene la posibilidad de que un cambio atencional encubierto, es decir, un cambio atencional en el que la mirada está fija y no involucra movimiento ocular alguno, tenga como consecuencia una diferencia fenomenológica. ¿Qué explicación podría dar un representacionalista sobre esta esta diferencia? El mismo Chalmers asegura que el representacionalismo debe considerarse como una clase de "hipótesis nula que sólo debe rechazarse si disponemos de evidencia fuerte en su contra" (2010, p. 349) y se aventura a realizar sugerencias respecto de cómo enfrentar este desafío sin evaluarlas críticamente o comprometerse con alguna. Por ejemplo, señala que el representacionalista podría afirmar que "la posición o el color de la luz a la cual uno se encuentra atendiendo es representada con mayor especificidad que la luz a la cual no se está atendiendo" (2010, p. 349) o que el punto lumínico a la cual se atiende es representado "como siendo más saliente que la otra luz" (2010, p. 349), aunque reconoce que no tenemos claridad alguna acerca de qué clase de propiedad podría ser la "saliencia" fenomenológica en caso de que exista.

Más allá de las consideraciones preliminares realizadas por Chalmers al evaluar el cambio atencional como algo que podría incomodar al representacionalismo es necesario detenerse y revisar la literatura psicológica para determinar si esto es efectivamente no solo una posibilidad, sino algo ya documentado en el dominio experimental. Por este motivo revisaremos a continuación evidencia que sí sugiere la existencia de variación fenomenológica como consecuencia de la implementación de la atención. Una vez corroborado empíricamente el desafío atencional veremos qué estrategias tiene el representacionalismo para enfrentar este problema y argumentaremos que una de ellas parece vislumbrarse como más prometedora.

\section{Antecedentes empíricos}

La filosofía contemporánea ha destacado el hipotético rol que posee la atención en los modelos de explicación reductivos y no-reductivos del surgimiento de la conciencia perceptiva $^{3}$. Sin embargo, sea o no la atención empíricamente necesaria y/o suficiente para la conciencia perceptiva, son pocas las discusiones filosóficas que han centrado su interés en los efectos que la atención causa en la fenomenología o carácter fenoménico

3 Distingo entre superveniencia y determinación por una motivación dialéctica. La primera es una variable metafísica y la segunda una variable de naturaleza explicativa. En estricto rigor si $x$ determina totalmente a $y, y$ necesariamente superviene en $x$. Me interesa enfatizar que en un enfoque purista en el que el carácter fenoménico está totalmente determinado por el contenido representacional no hay necesidad de incorporar en la explicación propiedades psicológicas no-representacionales, como podría ser la manera o modo en que representamos el mundo (Chalmers 2010, Crane 2003). 
de nuestras experiencias. Esto contrasta con el interés reciente que ha tenido este tópico al interior de la psicología. Existen numerosos estudios que verifican la hipótesis, según la cual la atención parece tener toda clase de efectos menores (que son reproducibles y estadísticamente relevantes) en la fenomenología de nuestras experiencias. Algunas investigaciones sugieren que la atención afecta la apariencia visual de propiedades como el contraste (Carrasco et al. 2004), el brillo aparente (Tse 2005), el tamaño aparente de objetos (Anton-Erxleben et al. 2007), la saturación aparente de un color (Fuller \& Carrasco 2006) o la distancia aparente entre dos objetos (Liverence \& Scholl 2011). Ilustremos esto con un caso paradigmático de la literatura que demuestra que la atención modifica significativamente el contraste aparente. El siguiente es el experimento realizado por Carrasco et al. $(2004)^{4}$ :

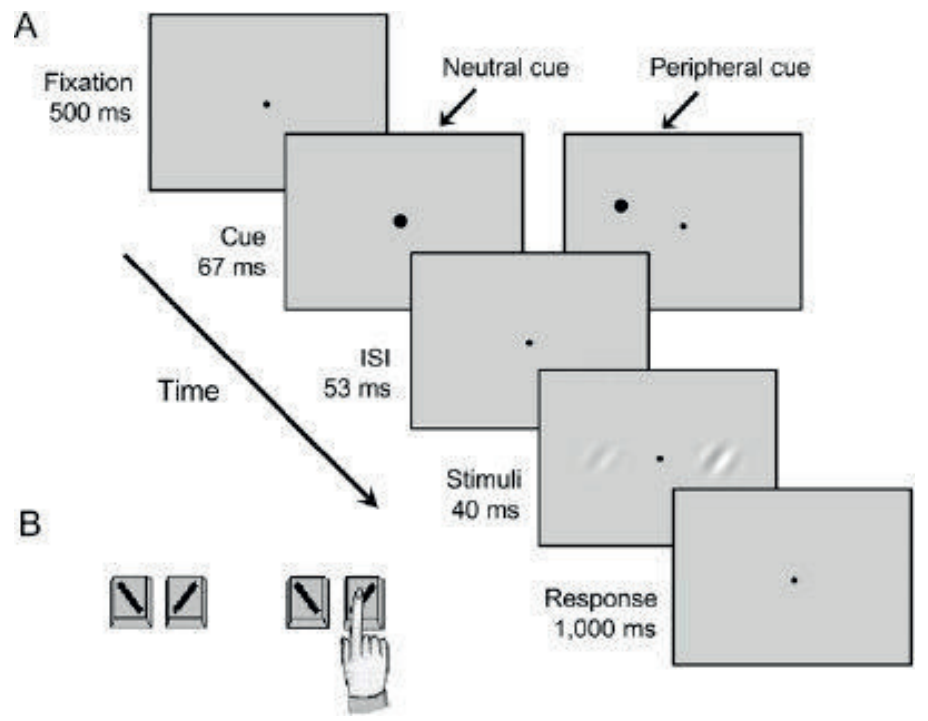

En este conocido experimento los participantes fijan su mirada en un punto específico por 0,5 segundos. Luego una pista es presentada por 67 milisegundos ya sea en el punto de fijación (pista neutral) o en una posición cercana en que aparecerá uno de los filtros de Gabor (pista periférica). Después de un intervalo entre estímulos (ISI) de 53 milisegundos, dos filtros de Gabor se manifiestan por 40 milisegundos. La tarea de los participantes es reportar la orientación del filtro de Gabor que parece tener el mayor grado de contraste dentro de un margen de 1 segundo. La clave ciertamente es que estos reportes dependen directamente del contraste aparente de los filtros de Gabor, es decir, en cómo el contraste de estos filtros se manifiesta subjetivamente a cada uno de los participantes. ¿Cuál es la relevancia de este experimento para nuestro tema de discusión? La clave está en los efectos que posee la atención, específicamente en cómo 
atender encubiertamente un filtro de Gabor tiene como consecuencia que este aparente tener un contraste mayor del que posee. Si el filtro de Gabor estándar tiene un 6\% de contraste, la atención aumenta el contraste aparente en un 2,5\%. Así, por ejemplo, al atender un filtro de Gabor con 3,5\% de contraste este parece ser similar a un filtro del $6 \% \mathrm{y}$ al atender un filtro con un contraste del $22 \%$ este parece tener un contraste un $6 \%$ mayor manifestándose como equivalente al de un filtro del $28 \%$ que no está siendo atendido de forma encubierta ${ }^{5}$ :

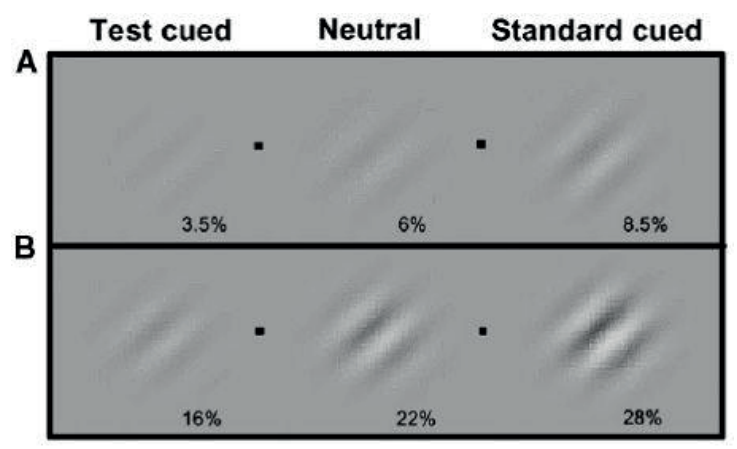

Un segundo cuerpo de evidencia que tiende a respaldar la hipótesis de que la atención afecta la apariencia de ciertas propiedades observables proviene de los estudios en torno al brillo realizados por Tse (2005). Utilizando imágenes como la que vemos a continuación los sujetos reportan que atender encubiertamente uno de los discos que se sobreponen en el panel de la izquierda (con fondo blanco), mientras se mantiene un punto de fijación ocular, tiene como efecto que este disco específico parezca ser más oscuro de lo que es realmente ${ }^{6}$. Esto no sucedería en el caso del panel de la derecha que posee un fondo negro. Tal como señala $\mathrm{Wu}$, “el punto aquí es que la atención parece afectar la fenomenología, en este caso disminuyendo el brillo aparente del disco atendido (o aumentando el contraste relativo entre los discos)" (2014, p. 122).
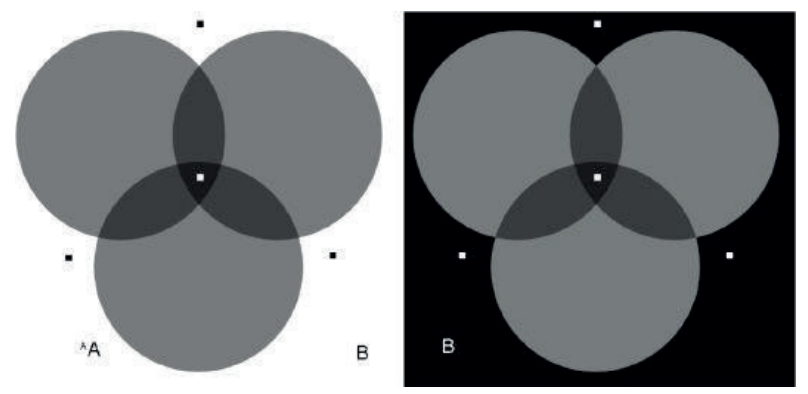

5 Imagen extraída para uso explicativo de Carrasco et al. (2004) "Attention Alters Appearance" Nature 7: 308-313.

6 Esta imagen también es original de Carrasco et al. (2004) "Attention Alters Appearance" Nature 7: 308-313. 
A diferencia del caso de Carrasco et al. (2004), aquí los sujetos pueden variar libremente el foco de atención entre los tres discos que se sobreponen en el panel de la izquierda sin los constreñimientos temporales asociados a la presentación de pistas en intervalos de solo algunos milisegundos. El efecto resultante podría caracterizarse como "ilusorio", ya que tendríamos una experiencia de brillo aparente que no se adecua a la de los ítems considerados de forma independiente ${ }^{7}$. No se trata simplemente de la posibilidad teórica de que un cambio atencional encubierto tenga como consecuencia una diferencia fenomenológica. Los reportes fundados introspectivamente sugieren que al menos en casos como los presentados esto es algo empíricamente verificable y no solo posible. El desafío para el representacionalismo es claro. Si el carácter fenoménico de una experiencia visual superviene y está determinado totalmente por su contenido representacional, entonces no puede haber modificación alguna de brillo o contraste aparente sin alteración del contenido representacional de la experiencia.

\section{Determinación y contenido representacional}

Parece evidente que la atención afecta las apariencias de un número importante de propiedades observables. En la sección anterior discutimos los experimentos de Carrasco et al. (2004) que ilustran cómo la atención encubierta modificaría el contraste aparente de un conjunto de filtros de Gabor cuando estos son observados en una tarea en la que se mantiene un punto de fijación. Uno de los resultados más sugerentes es el que se obtiene cuando se atiende un filtro con un contraste del $22 \%$ y éste se experimenta como si fuese equivalente al de un filtro Gabor del 28\% que no es atendido de forma encubierta. Aquí la alteración fenomenológica como efecto de la atención constituye un incremento del $6 \%$. Llamemos al filtro de Gabor que tiene un $22 \%$ de contraste $G 22$ y a la experiencia que tiene un sujeto al atenderlo encubiertamente Exp1. Ahora bien, si en este caso el sujeto mueve su atención solo dos grados a la derecha de $G 22$ y atiende el punto de fijación tendrá una experiencia con una fenomenología diferente en la que G22 aparenta tener un contraste menor. Refirámonos a esta segunda experiencia como Exp2. En Exp1 G22 aparenta tener un contraste del 28\%, mientras que en Exp2 su contraste aparente es del 22\%. Asimismo, durante Exp1, G22 aparenta tener un contraste idéntico al del filtro de Gabor G28 no atendido, que supuestamente posee un contraste de $28 \%$.

¿Cómo explica el representacionalismo esta alteración? Chalmers sugiere que lo atendido es representado "con mayor especificidad" (2010, p. 349) o "como siendo más saliente" (2010, p. 349). Su hipótesis es que la atención (o el grado en que atendemos un estímulo) aumenta la resolución o especifidad con que una propiedad es representada. Hay dos ventajas que parece tener esta lectura si analizamos el caso Carrasco et al. (2004). Primero, la diferencia fenomenológica existente entre Exp1 y Exp2 se explica por completo en términos de una diferencia representacional. Segundo,

Imagen originalmente publicada en Tse (2005) "Voluntary attention modulates the brightness of overlapping transparent surfaces". Vision Research 45: 1095-8. 
tanto los contenidos de Exp1 como Exp2 son correctos en el sentido de que no parecen ser representaciones "ilusorias" respecto de cómo son las cosas.

Me parece fundamental detenerse en el segundo aspecto, es decir, en la idea de que tanto el contenido de Exp1 como el contenido Exp2 son correctos o no ilusorios. ¿Cómo es esto posible? Parece intuitivo o de sentido común pensar que al menos uno de estos contenidos representacionales es incorrecto. La conclusión que Carrasco et al. (2004) sostienen es que la atención focal aumenta el contraste aparente de un estímulo. Por ello Exp1 y Exp2 representan a G22 como teniendo contrastes aparentes diferentes y durante Exp1 los filtros G22 y $G 28$ se experimentan como teniendo el mismo contraste. Dos consecuencias adicionales serían que el filtro de Gabor no atendido se vería fenomenológicamente empobrecido y que al comparar dos filtros que tienen el mismo contraste, los sujetos siempre juzgarían, en base a las apariencias, que el filtro de Gabor atendido tiene un mayor contraste. Por este motivo, es controvertido señalar que el contenido de ambas experiencias es correcto y parece sensato insistir en que la "saliencia" que resulta de la atención focal a G22 es ilusoria.

La intuición inmediata sería pensar, tal como sugerien Carrasco et al. (2004), que algunos de estos juicios son falsos porque se fundan en representaciones incorrectas. Sin embargo, autores como Nanay (2010) o Stazicker (2011a, 2011b) apuntan en una dirección muy diferente en la que los juicos y las representaciones subyacentes no tienen por qué ser incorrectos. La estrategia se funda en la distinción fundamental entre propiedades representacionales indeterminadas (determinables) y propiedades representacionales determinadas. Su tesis es que los contenidos de Exp1 y Exp2 son indeterminados, donde representar algo de modo indeterminado se debe entender como "representarlo como instanciando una propiedad determinable, sin compromiso respecto de qué determinación de aquella propiedad determinable instancia. A grandes rasgos, la propiedad $A$ determina la propiedad $B$ donde tener $A$ es tener $B$ de una manera específica" (Stazicker 2011b, p. 170). Por ejemplo, uno podría representar algo como siendo rojo, sin representarlo como siendo una determinación específica de ser rojo (amaranto, carmesí, escarlata, bermellón, granate, etc.). Si esto es así la representación en cuestión sería indeterminada o determinable en el sentido expuesto anteriormente.

La motivación de esta estrategia es que al ser indeterminadas nuestras representaciones, los juicios que se fundan en ellas no tienen por qué ser incorrectos. Si tú representas el mantel que cubre la mesa de comedor como siendo azul cobalto, tu representación será incorrecta si de hecho el mantel es azul marino. Pero si tu representas el mantel como siendo simplemente azul, tu representación será correcta independientemente de si el mantel es específicamente azul cobalto o azul marino. Es decir, entre más indeterminada sea tu representación del color del mantel más amplia será la gama de colores específicos que éste puede tener que sean consistentes con el hecho de que tu representación sea correcta.

Lo mismo sucedería hipotéticamente en el caso específico de los experimentos de Carrasco et al. (2004). Los contenidos de Exp1 y Exp2 serían equivalentes a rangos de indeterminación que incluirían el contraste objetivo que posee cada uno de los filtros Gabor (22\% y $28 \%$ ) y en este sentido, correctos. Bajo la lectura de Stazicker 
(2011a, 2011b, 2018) y Nanay (2010), Exp1 y Exp2 representan un rango de contrastes indeterminado. Por ejemplo, Exp1 podría representar el rango indeterminado de contrastes entre el $15 \%$ y el $23 \%$, mientras que Exp2 representaría indeterminadamente el rango entre el $21 \%$ y el $29 \%$. El contenido representacional de ambas experiencias sería verídico respecto de los contrastes de los filtros de Gabor percibidos (22\% y $28 \%$ ), debido a que los rangos de representación indeterminada en juego serían compatibles con las propiedades de contraste determinadas que poseen objetivamente los filtros (Watzl 2019, p. 591).

Esta propuesta representacionalista fundada en la existencia de propiedades indeterminadas ha recibido diversas objeciones. Block, por ejemplo, sostiene que si aceptamos que los contenidos representacionales en juego son indeterminados, "la indeterminación del contenido tendría que verse reflejada en una indeterminación del look" (2010, p. 52). En otras palabras, la fenomenología tendría que exhibir de alguna $\mathrm{u}$ otra forma el rango de indeterminación que caracteriza a los contenidos. Por una parte, la objeción enfatiza una supuesta inadecuación fenomenológica de la propuesta sustentada en que la forma en que experimentamos conscientemente los estímulos que atendemos, en este caso los filtros Gabor, en ningún caso es vaga o indeterminada. Todo lo contrario, durante estas experiencias nos parece que los estímulos poseen un look único o específico. Por otra parte, Block agrega que no hay algo así como un look único o específico que pueda reflejar un rango de indeterminación como el propuesto.

El punto es que no hay un "look" único que algo tiene si es que tiene un contraste del $22 \%$ más o menos un $6 \%$. Por analogía, consideren la suposición de que algo luce de la siguiente manera: rectangular o triangular o circular. Aquel predicado disyuntivo no describe el look que algo puede tener, al menos no en circunstancias perceptuales normales (Block 2010, p. 52).

Respecto a estas objeciones, Stazicker (2011a, 2018) tiene algunas observaciones a mi juicio pertinentes. Block sugiere que si el contenido es indeterminado, esto se debería ver reflejado en el carácter fenoménico de la experiencia. Sin embargo, parece perfectamente plausible sostener que en algunos casos algo puede ser representado de manera indeterminada sin que la fenomenología sea poco específica o detallada. Pensemos, a modo de ejemplo, en algunas propiedades como el largo, el ancho o la altura de un objeto. Parece adecuado afirmar que un objeto puede ser representado como instanciando la propiedad determinable de tener entre 1 y 2 metros de altura o entre 25 y 50 centímetros de ancho. El contenido en este caso es indeterminado, pero en ningún caso el carácter fenoménico de esta experiencia es caracterizado como vago, indeterminado o difuso.

Además, los objetos que en estos casos están siendo representados de manera indeterminada no parecen tener un look más detallado o específico. Uno podría afirmar, sobre la base del carácter fenoménico de una experiencia a la distancia, que una persona parece medir entre $1.78 \mathrm{~m}$ y $1.83 \mathrm{~m}$ de altura sin pensar que la fenomenología de mi experiencia es más indeterminada o específica de lo que afirmo. Block supone que introspectivamente uno siempre puede establecer el rango de indeterminación de un contenido experiencial, pero esto no parece ser el caso. Es muy difícil discriminar introspectivamente entre dos experiencias que poseen contenidos determinables 
diferentes (Stazicker 2018). Si esto es así, las objeciones de Block no parecen del todo concluyentes.

Lo anterior no significa que la estrategia fundada en la representación indeterminada esté a salvo. Watzl (2019) ha formulado un problema que a mi juicio debe considerarse con máxima detención. En los casos mencionados hemos hablado de contenidos qua rangos de indeterminación compatibles. Exp1 representa el rango indeterminado de contrastes entre el 15\% y el 23\%, mientras que Exp2 representa indeterminadamente el rango de contrastes entre el $21 \%$ y el $29 \%$. Estos contenidos son compatibles, es decir, se superponen o incluyen un parámetro de indeterminación (21\% al 23\%) que nos permite hablar de corrección o veracidad en ambos casos.

Sin embargo, es fácil concebir un escenario posible en el que las propiedades indeterminadas representadas por las experiencias son incompatibles (no se superponen):

Supongan, por ejemplo, que de alguna forma propiedades de contraste más determinadas son representadas, tal que E1 representa el 24-28 por ciento mientras que E2 el 18-22 por ciento. Este escenario... es compatible con el cambio empíricamente observado de contraste aparente por 6 puntos porcentuales y, sin embargo, las propiedades determinables representadas por E1 y E2 no se superponen, tal que al menos una de ella debe ser incorrecta (Watzl 2019, p. 592).

Un escenario donde los rangos de indeterminación no se superponen sería suficiente para generar un problema de arbitrariedad. ¿Qué tipo de problema de arbitrariedad? El de no saber si estamos ante un caso en el que los contenidos indeterminados de Exp1 y Exp2 se superponen o no. Y si los contenidos indeterminados no se superponen sería arbitrario afirmar que el contenido de Exp1 es el correcto, mientras que el de Exp2 no lo es (o al revés). La posibilidad de solo un escenario de incompatibilidad es suficiente para cuestionar al menos en parte las pretensiones de la estrategia fundada en la indeterminación y no disponemos hoy de evidencia empírica alguna que nos permita zanjar esta cuestión (Watzl 2019, p. 593).

Las consideraciones anteriores tienen implicancias relevantes a la hora de evaluar la estrategia basada en la idea de contenidos representacionales qua rangos de indeterminación. Ésta parece estar en lo cierto al afirmar que la atención afecta el grado de determinación con que experimentamos ciertas propiedades. Experimentamos aquello que atendemos con un mayor grado de determinación o detalle. Sin embargo, hay dos consecuencias adicionales que son controvertidas y problemáticas. Primero, si bien en ocasiones representamos propiedades determinables, es dudoso que esto siempre sea así como se insinúa. Me imagino, por ejemplo, que un experto en discriminar e identificar ciertas clases de propiedades sensibles - un catador de vinos, un barista, un especialista en teoría del color-será capaz de experimentarlas siempre con el más alto grado de determinación a diferencia del no experto. Segundo, la posibilidad de escenarios representacionales que no se superponen revive la intuición inicial de que al menos uno de los contenidos que representan los filtros de Gabor en los casos de Carrasco et al. (2004) debe ser ilusorio. Pero incluso si estamos dispuestos a abrazar esta intuición no parece existir aún un criterio no-arbitrario para distinguir en estos casos cuál es la representación incorrecta. 


\section{IV. ¿Una ilusión masiva?}

Una estrategia representacionalista completamente diferente para enfrentar el desafío atencional ha sido propuesta por Watzl (2019). Es una propuesta radical, ya que renuncia por completo a la ausencia de error o precisión (accuracy) como criterio fundamental para evaluar qué sucede cuando la atención altera la apariencia de ciertas propiedades. Tanto la Expl en que representamos a G22 con un contraste aparente del 28\%, como la Exp2 en que lo representamos con un contraste aparente del 22\%, serían experiencias cuyo contenido representacional es impreciso. La invitación de Watzl (2019) es a adoptar una forma de representacionalismo donde siempre hay una suerte de ilusión masiva sustentada en una distinción funcional esencial entre percibir y atender. A su juicio, "mientras que la función central de los sistemas perceptuales es facilitar representaciones precisas de nivel personal del entorno del organismo, la función central de la atención perceptual es hacer que estas representaciones de nivel personal sean útiles" (Watzl 2019, p. 597).

¿En qué se sustenta esta divergencia funcional y cómo contribuiría a solucionar el desafío atencional? En el ámbito de las ciencias cognitivas hay diversas posiciones respecto a la relación existente entre conciencia perceptual y atención ${ }^{8}$. Watzl ciertamente está en la posición extrema según la cual atención y percepción podrían considerarse, al menos desde una perspectiva funcional, como capacidades diferentes. Por un parte, la percepción visual tendría una función esencialmente representacional. Nuestras capacidades perceptuales -al igual que las capacidades perceptuales de otros organismos- facilitan la formación de representaciones de una amplia gama de propiedades distales que se encuentran en el ambiente del organismo9. Por otra parte, la función de la atención sería la de priorizar selectivamente ciertos estímulos sobre otros tal que el organismo utilice esta información específica para otros fines como podrían ser la acción (Wu 2011) o el control racional del pensamiento y la acción (Smithies 2011). Para Watzl (2019), la percepción visual tiene la función de representar con precisión propiedades del entorno y la atención, la de facilitar que esta información representada sea utilizable. La clave de su propuesta sería que hay ocasiones específicas en que el

8 En adelante utilizaré la expresión "contraste aparente" para referirme al contraste tal como lo experimentamos en el dominio fenomenológico. Hablaré simplemente de "contraste" sin mayores calificaciones o "contraste objetivo" cuando me refiera a la propiedad que por hipótesis un ítem del entorno posee independientemente de si lo estamos percibiendo o atendiendo.

9 En el ámbito de la filosofía de las ciencias cognitivas normalmente es posible distinguir al menos cinco posiciones respecto de la relación precisa que existe entre conciencia perceptual y atención (Taylor 2013): (i) La atención es necesaria y suficiente para la conciencia (De Brigard \& Prinz 2010: Prinz 2011, 2012), (ii) la atención es necesaria, pero no suficiente para la conciencia (Cohen et al. 2012), (iii) la atención es suficiente, pero no necesaria para la conciencia (Smithies 2011, Mole 2008), (iv) la atención no es suficiente para la conciencia (Norman et al. 2013, Mole 2014), y (v) la atención no es necesaria ni suficiente para la conciencia (Lamme 2003, 2010; Koch \& Tsuchiya, 2007). 
organismo necesita sacrificar una de estas funciones en privilegio de la otra, "ya que la representación más útil podría no ser la más precisa y la más precisa podría no ser la más útil” (Watzl 2019, p. 598).

Los estudios de Carrasco et al. (2004) podrían explicarse en estos términos. El organismo sacrificaría la precisión o adecuación de las representaciones con el fin de enfatizar atencionalmente algunos rasgos relevantes del estímulo. Por ello algunos creen que "la atención aumenta la percepción... enfatizando detalles relevantes a expensas de la representación fiel de una entrada sensorial" (Carrasco Fuller \& Ling 2008, p. 1162). En el caso discutido podríamos pensar que la atención afecta la fenomenología aumentando el contraste aparente del estímulo para que la representación sea más útil. La hipótesis en este contexto sería que la representación más útil no es necesariamente la más precisa, aunque evidentemente hay que estar abierto a situaciones en que la precisión de la representación sería funcional a la utilidad ${ }^{10}$.

La estrategia de la divergencia funcional es atractiva, pero conlleva algunas dificultades difíciles de aceptar para importantes paradigmas teóricos y experimentales. En primera instancia, cualquier tipo de diferenciación funcional entre percibir y atender acarrea serios problemas para aquellas teorías en las que la atención es empíricamente suficiente para la conciencia perceptiva, postura estándar para gran parte de las ciencias cognitivas hoy (Prinz 2012). Pero aún más controvertido me parece aseverar que nuestra capacidad para representar perceptualmente propiedades como el contraste, el color o la saturación siempre conlleva un grado de error o ilusión, aunque sea leve. ¿Por qué? Aquí hay una incomodidad explicativa y otra estrictamente epistémica. En términos explicativos, la tesis de una ilusión masiva atenta contra cualquier forma de naturalismo filosófico que intente dar cuenta del origen de nuestras habilidades representacionales en términos de rastreo o covariación causal confiable. Esto es un problema serio, a no ser que deseemos aceptar la posibilidad de que existan representaciones confiables pero erróneas (Mendelovici 2013).

Por último, normalmente se afirma que atender un objeto/propiedad en el entorno mejora la situación epistémica en que nos encontramos. Los juicios que realizamos acerca de esos objetos/propiedades tendrían mayor justificación si de hecho los atendemos. No quedaría del todo claro cómo es que existe un beneficio epistémico asociado al grado de justificación que tenemos para creer cuando atendemos si la atención funcionalmente no está esencialmente ligada al grado de adecuación o precisión de nuestras representaciones. Las consideraciones anteriores quizás no son definitivas, sobre todo para las teorías que no pretenden naturalizar el origen de nuestras capacidades representacionales, pero efectivamente son a mi juicio suficientes para motivar una alternativa.

10 Esto está presente en diversas propuestas científicas como la de Marr (1982) o filosóficas como la de Burge (2010). 


\section{Una propuesta narcisista}

Todas las propuestas anteriores asumen una perspectiva clásica acerca de cómo y qué es representado durante una experiencia. La mirada clásica supone que nuestros sentidos tienen la función de generar representaciones correctas y precisas acerca del mundo, además de confiar en que este último está constituido de propiedades intrínsecas cuya existencia no depende de nuestros actos perceptivos. Akins (1996) señala que esta perspectiva clásica incluye la idea de que nuestros sistemas sensoriales tienen tres atributos centrales. Primero, son sistemas orientados a la veracidad/adecuación: cada señal o representación está correlacionada con alguna propiedad objetiva en el mundo. A modo de ejemplo, hemos discutido en las secciones anteriores hasta qué punto los contenidos de nuestras experiencias son correctos o si las apariencias se adecuan a cómo las cosas son. Segundo, los sistemas sensoriales preservan la estructura de las diversas relaciones que existen entre propiedades externas codificando esta información de manera sistemática. Nuestras representaciones serían un fiel espejo de las relaciones externas. Finalmente, nuestros sistemas sensoriales representan sin ficción alguna (cambio, adorno, embellecimiento, exageración) ítems en el mundo externo.

Es muy relevante preguntarse si la interpretación clásica realmente aplica a todos los sistemas sensoriales y a todas las propiedades que estos representan. Akins (1996) planteó esta pregunta al estudiar cómo opera el sistema termorreceptor en nuestro organismo y llegó a la conclusión de que esto no es así siempre. Las sensaciones termales particulares no están necesariamente correlacionadas con ninguna temperatura o cambio de temperatura en particular. Tampoco estas sensaciones reflejan la estructura de relaciones existentes entre estímulos térmicos externos y en muchos casos el sistema termorreceptor genera sensaciones claramente exageradas (Akins 1996, pp. 351-352), por ejemplo, al sumergir la cabeza en agua helada o al tocar con la punta de los pies el agua caliente antes de un baño.

Los estudios en torno a la termorrecepción motivan consideraciones filosóficas importantes para la funcionalidad de otros sistemas. La primera es que en algunos casos los sistemas sensoriales no han evolucionado con el objetivo de responder "¿Cómo es allá afuera? -una pregunta acerca de la temperatura objetiva..." (Akins 1996, p. 349), sino para dar cuenta de cómo son las cosas relativas a nuestros intereses. En este sentido, el paradigma clásico que privilegia la formación de representacionales precisas $o$ verídicas que reflejan cómo son las propiedades externas e independientes no sería el dominante. Más bien, algunos sistemas como el termorreceptor serían "narcisistas" (Akins 1996, Prettyman 2017) al privilegiar cómo es el entorno para el organismo que percibe y de acuerdo con sus necesidades.

Otra consideración filosófica dice relación ya no con la función, sino con la naturaleza de las propiedades que estos sistemas representan o detectan. Por una parte, habría propiedades que son objetivas en un sentido clásico, es decir, que no varían de modo alguno con las modificaciones o cambios que pueda sufrir el organismo que las percibe. Las llamadas cualidades primarias como la forma o el tamaño son ejemplos. Por otra parte, existirían propiedades como el gusto, el sabor o la temperatura que satisfacen el carácter narcisista expuesto en las observaciones de Akins (1996). Serían 
propiedades relacionales cuya existencia no está determinada solo por el mundo, sino también por los intereses y necesidades de los organismos.

La investigación que Prettyman (2017) ha elaborado frente al desafío atencional se sitúa bien en este marco relacional/narcisista, ya que enfatiza dos cuestiones que nos interesan: (i) la atención altera la fenomenología de nuestras experiencias y (ii) esto puede modelarse en términos representacionales sin sacrificar completamente la idea de veracidad o precisión. El contraste aparente de un estímulo mientras es atendido sería un ejemplo de propiedad relacional/narcisista que está indexada a una variable del organismo que representa. La variable en este caso es el grado de atención que se implementa a un estímulo, por lo que el contraste aparente de un filtro de Gabor como los utilizados en los experimentos de Carrasco et al. sería una propiedad indexada a la atención del organismo.

Mientras atendemos encubiertamente el filtro de Gabor G22 (Expl) este aparenta tener un contraste del $28 \%$, mientras que al quitarle la atención su contraste aparente es solo del 22\% (Exp2). La interpretación clásica nos dispone a pensar que al menos una de estas experiencias -Exp1 o Exp2- posee un contenido representacional incorrecto. ¿Por qué? Porque el paradigma clásico supone que el contraste aparente que caracteriza la fenomenología de nuestras experiencias debe adecuarse con precisión a cómo es cada filtro de Gabor de manera intrínseca, sin considerar los intereses y necesidades del sujeto que percibe.

Es en este contexto donde el proyecto narcisista sugiere un punto de partida claramente diferente. Imaginen que dos personas - A y B-ingresan a una habitación que según un termostato electrónico se encuentra a $25^{\circ} \mathrm{C}$. Supongan que A viene del exterior donde hay sólo $5^{\circ} \mathrm{C}$, mientras que $\mathrm{B}$ viene de la habitación contigua que se encuentra a $30^{\circ} \mathrm{C}$. Claramente las sensaciones térmicas de ambos serán diferentes, ya que estarán indexadas al estado interno en que se encuentran los organismos. ¿Estaríamos dispuestos a afirmar sin tapujos que la fenomenología de al menos una de estas experiencias térmicas -ExpA y ExpB- es incorrecta o ilusoria? Me parece que no. Yo estaría inclinado a afirmar que lo que A y B sienten refleja la estructura relacional de las propiedades diferentes que figuran en los contenidos de sus experiencias. Lo relevante aquí no es si lo que ellos sienten es preciso respecto de la temperatura ambiente, sino más bien si contribuye a las necesidades o intereses del organismo (abrigarse, desabrigarse, etc.).

Algo similar pienso es factible afirmar de las diferencias fenomenológicas que surgen con la variable atencional, ya que en ellas se exhibe el resultado de un proceso en que los sistemas (la visión) "codifican información de tal forma que relacionan el objeto, propiedad o evento al estado relevante del sujeto" (Prettyman 2017, p. 4044). Tal como las propiedades térmicas de una habitación se codifican en relación con un conjunto de estados internos de A y B, las propiedades de los filtros de Gabor en los experimentos de Carrasco et al. (2004) se codifican "narcisísticamente" en relación con la dirección de la atención. Un filtro de Gabor G22 puede gatillar la manifestación de contrastes aparentes diferentes dependiendo de si lo atendemos o no. Nuestras experiencias reflejan así la dirección de la atención y no una estructura inherente al filtro que está siendo observado. Prettyman (2017) sugiere que al atender G22 la codificación 
narcisista sería $28 \%$-atendido-por-mí, mientras que cuando experimentamos sin atender G28 la codificación narcisista sería 28\%-no/atendido-por-mí. Además, no tendríamos que renunciar a la veridicalidad o precisión de Exp1 y Exp2, ya que las propiedades relacionales representadas son diferentes.

Watzl (2019, p. 594) ha afirmado que esta lectura relacional es problemática por dos razones. Primero, habría evidencia psicológica suficiente como para afirmar que las experiencias de contraste que tenemos son invariables bajo una amplia variedad de condiciones (Georgeson \& Sullivan 1975), indicando así que las propiedades en cuestión no serían relacionales. No creo que esto sea una dificultad para el proyecto de Prettyman (2017). Ella reconoce que hay razones para pensar que hay constancia fenomenológica ante condiciones/contextos variables como el grado de iluminación del estímulo o la distancia perceptual, pero lo que está en juego aquí es un contexto atencional y una de las lecciones de Carrasco et al. (2004) es que en estos contextos no hay constancia fenomenológica.

La segunda objeción contra el narcisismo apela al fenómeno de la comparación entre contrastes. Es usual comparar entre los contrastes aparentes de dos experiencias diferentes que involucran diferentes grados de atención. En nuestro caso predilecto, el contraste aparente que exhibe $G 22$ durante $\operatorname{Exp} 1$ (28\%) es diferente al que experimentamos en $\operatorname{Exp} 2$ (22\%). El proyecto narcisista explica la diferencia fenomenológica sobre la base de una diferencia de las propiedades relacionales (propiedades indexadas al grado de atención implementado) que cada una de las experiencias representa. Ahora bien, hay una pregunta interesante: ¿Qué sucede cuando atendemos $G 22$ y este aparenta tener el mismo contraste que $G 28$ que se encuentra desatendido a la derecha del punto de fijación? Aquí Watzl objeta y señala que es razonable juzgar que "si dos ítems parecen tener el mismo contraste, entonces hay algún contraste que ambos ítems parecen tener" (2019, p. 594). De acuerdo con la propuesta narcisa/relacional el desafío atencional se explica en términos de una diferencia de las propiedades representadas. Sin embargo, Watzl sugiere que esto es inconsistente con la indiscriminabilidad subjetiva de los contrastes aparentes de los dos filtros, la cual exigiría sostener que estos ítems diferentes de hecho comparten el mismo contraste aparente.

A mi juicio, Watzl comete un error importante al elaborar su objeción sobre la base de una usual confusión entre consideraciones estrictamente epistemológicas y compromisos metafísicos. Una cuestión es la indiscriminabilidad entre el contraste aparente del ítem atendido y el ítem desatendido. Otra cuestión es la naturaleza de las propiedades que figuran en el contenido representacional de nuestra experiencia. Esto que Watzl prefiere no discutir o estratégicamente ignora es básico para toda teoría relacional de la percepción. Si queremos especificar la estructura metafísica de nuestras experiencias perceptuales y sus contenidos representacionales no tenemos por qué fundar nuestras reflexiones en la indiscriminabilidad. No se sigue del hecho de que dos ítems aparenten tener el mismo contraste, que estos de hecho tengan algo así como un contraste en común o un "máximo común denominador" (McDowell 1994). En otros términos, creo que no hay razón alguna para transitar de un hecho epistémico negativo como la indiscriminabilidad entre dos propiedades a una afirmación metafísica como la supuesta identidad de propiedades. 
Hemos afirmado que para el representacionalismo la fenomenología de una experiencia superviene y está totalmente determinada por su contenido representacional. Estrictamente hablando, el proyecto narcisista/relacional impulsado por Prettyman (2017) es plenamente consistente con esta idea. Lo fundamental es que no pueden existir alteraciones fenomenológicas como las implicadas por el desafío atencional, sin alteración alguna en las propiedades representacionales de las experiencias. Esto deja abierta la posibilidad de que experiencias que poseen contenidos representacionales diferentes sean de hecho indiscriminables en el dominio de las apariencias ${ }^{11}$.

\section{Consideraciones finales}

Durante años se consideró como potencial problema para el representacionalismo la posibilidad teórica de casos en que nuestra fenomenología se modificara considerablemente sin que esto implicara un cambio evidente a nivel del contenido representacional. Esta posibilidad teórica se ha confirmado empíricamente en estudios como los de Carrasco et al. (2004) que ilustran cómo la atención encubierta genera cambios fenomenológicos en propiedades como el contraste aparente. Ante este tipo de desafíos, el representacionalismo tiene la posibilidad de traicionar sus compromisos tradicionales apelando a la existencia de propiedades psicológicas intrínsecas (no-representacionales) que expliquen estas alteraciones o entregarse a la difícil tarea de conservar la relación de superveniencia y determinación total entre carácter fenoménico y contenido representacional como la única relevante. Aquí hemos adoptado la segunda estrategia mediante una evaluación crítica de tres alternativas que no están libres de las dificultades internas inherentes a todo proyecto filosófico.

Para responder al desafío atencional consideramos primero la posibilidad de comprender la forma en que nuestras experiencias representan el entorno en términos de rangos indeterminados de valores y no mediante la especificación de un valor determinado. Más allá de una potencial inadecuación fenomenológica (normalmente no parecemos experimentar subjetivamente rangos indeterminados), esta interpretación conlleva la controvertida tarea de corroborar empíricamente de un modo no arbitrario cuando es pertinente juzgar que estamos de hecho ante una experiencia que representa correctamente propiedades como el brillo o el contraste aparente. En segunda instancia, se evaluó la estrategia de distinguir entre percepción y atención bajo la división funcional existente entre representar con precisión el entorno y hacer que la información representada sea útil. Una de las consecuencias controvertidas de este modelo es que las alteraciones fenomenológicas que resultan de la atención se conciben como testimonio del hecho de que en estos casos sacrificamos la precisión a favor de la utilidad. Nuestras experiencias serían útiles, pero siempre en un mayor o menor grado ilusiones. Lo anterior no solo conlleva serios problemas para aquellos modelos teóricos que sostienen que la atención

11 Otro ámbito en el que podríamos contrastar entre utilidad y adecuación representacional es el caso de las constancias perceptuales de algunas propiedades observables. 
es empíricamente necesaria y/o suficiente para la conciencia perceptual, sino que también pone en jaque algunos de los supuestos básicos del naturalismo filosófico que intenta explicar el origen de nuestras representaciones bajo un modelo de covariación fiable.

Por último, analizamos la viabilidad de un novedoso proyecto filosófico que cuestiona algunos de los compromisos clásicos de las teorías perceptuales para dar pie a un marco teórico ecológico y relacional para una gama acotada de propiedades representacionales. Motivados por las investigaciones de Akins (1996) acerca del funcionamiento de algunos sistemas sensoriales como el sistema termorreceptor y el trabajo filosófico de Prettyman (2017) se sostuvo que hay buenas razones para pensar que propiedades discutidas en los trabajos experimentales de Carrasco et al. (2004) son narcisistas o relacionales, ya que sufren modifican en consonancia con los intereses y necesidades del organismo. En el caso específico del contraste aparente, se trataría de una propiedad indexada al grado de atención que se implementa. El narcisismo, tal como lo entiende Prettyman (2017), es por el momento un proyecto a mi juicio prometedor que merece mayor atención y que posee algunas virtudes comparativas evidentes que lo hacen preferible a otras variantes del representacionalismo. Es una estrategia consistente con la asimetría implicada por la relación de superveniencia y determinación total existente entre carácter fenoménico y contenido representacional, preserva compromisos mínimos de adecuación respecto de las propiedades que están siendo representadas, acentúa la utilidad que caracteriza funcionalmente a los mecanismos atencionales y preserva una lección fundamental sin comprometernos con una ilusión masiva: la atención altera la fenomenología de nuestras experiencias.

\section{Referencias bibliográficas}

Akins, K. (1996), “Of sensory systems and the "aboutness" of mental states", Journal of Philosophy 93 (7): 337-372.

Anton-Erxleben, K., C. Henrich and S. Treue (2007), “Attention changes perceived size of moving visual patterns", Journal of Vision 7 (11): 5, 1-9.

Block, N. (2010), “Attention and mental paint”, Philosophical Issues 20 (1): 23-63.

Burge, T. (2010), Origins of Objectivity. Oxford: Oxford University Press.

Carrasco, Marisa, Stuart Fuller and Sam Ling (2008), “Transient Attention Does Increase Perceived Contrast of Suprathreshold Stimuli: a Reply to Prinzmetal, Long, and Leonhardt (2008)", Perception \& Psychophysics 70 (7): 1151-64.

Carrasco, M., S. Ling and S. Read (2004), "Attention alters appearance", Nature Neuroscience 7 (3): 308-13.

Chalmers, D. (2010), "The Representational Character of Experience", in D. Chalmers, The character of consciousness. Oxford: Oxford University Press, pp. 339-379.

(2006), "Perception and the Fall from Eden", in T. Gendler \& J. Hawthorne, ed., Perceptual experience. Oxford: Oxford University Press. 
Cohen, M.A., P. Cavanagh, M. Chun and K. Nakayama (2012), "The attentional requirements of consciousness", Trends In Cognitive Sciences 16 (8): 411-417.

Crane, T. (2003), The intentional structure of consciousness, in Q. Smith and A. Jokic, eds., Consciousness: New Philosophical Perspectives. Oxford: Oxford University Pres.

De Brigard, F. \& J. Prinz (2010), “Attention and consciousness”, Wiley Interdisciplinary Reviews: Cognitive Science 1: 51-59.

Dretske, F. I. (1995), Naturalizing the Mind. The MIT Press.

Fuller, S., and M. Carrasco (2006), Exogenous attention and color perception: Performance and appearance of saturation and hue, Vision Research 46: 40324047.

Georgeson M. A. \& G. D. Sullivan (1975), Contrast constancy: deblurring in human vision by spatial frequency channels, J. Physiol 252: 627-656.

Koch, C. and N. Tsuchiya (2007), "Attention and consciousness: two distinct brain processes", Trends in Cognitive Sciences 11 (1): 16-22.

Lamme, V. (2010), "How neuroscience will change our view on consciousness", Cognitive Neuroscience 1 (3): 204-240.

(2003), "Why visual attention and awareness are different", Trends in Cognitive Sciences 7: 12-18.

Liverence, B. M. and B. J. Scholl (2011), "Selective Attention Warps Spatial Representation: Parallel but Opposing Effects on Attended Versus Inhibited Objects", Psychological Science 22 (12): 1600-1608.

Lycan, W. (1996), Consciousness and Experience. Cambridge: MIT Press.

Marr, D. (1982), Vision. A Computational Investigation into the Human Representation and Processing of Visual Information. Freeman.

McDowell, J. (1994), Mind and world. Cambridge, Mass.; London: Harvard University Press.

Mendelovici, A. (2013), "Why tracking theories should allow for clean cases of reliable misrepresentation", Disputatio 8 (42): 57-92. 2016.

Mole, C. (2014), “Attention to Unseen Objects", Journal of Consciousness Studies 21, No. 11-12: 41-56.

(2008), "Attention and consciousness", Journal of Consciousness Studies, 15 (4): 86-104.

Nagel, T. (1974), “What Is It Like to Be a Bat?”, Philosophical Review 83: 435-50.

Nanay, B. (2010), “Attention and perceptual content”, Analysis 70 (2): 263-270.

Norman, L. J., C. A. Heywood and R.W. Kentridge (2013), “Object-Based Attention Without Awareness", Psychological Science 24 (6): 836-43.

Prettyman, A. (2017), "Perceptual Content is Indexed to Attention", Synthese 194 (10): 4039-4054.

Prinz, J. (2012), The Conscious Brain. Oxford: Oxford University Press. 
(2011), "Is Attention Necessary and Sufficient for Consciousness?" en C. Mole, D. Smithies \& W. Wu, eds., Attention: Philosophical and Psychological Essays (2011). Oxford: Oxford University Press, pp. 174-203.

Smithies, D. (2011), “Attention Is Rational-Access Consciousness", en C. Mole, D. Smithies \& W. Wu, eds., Attention: Philosophical and Psychological Essays Oxford: Oxford University Press, pp. 247-273.

Stazicker, J. (2018), "The visual presence of determinable properties", Phenomenal Presence, Dorsch, F. \& F. Macpherson, eds. Oxford: Oxford University Press, pp. 105-133.

(2011b), "Attention, visual consciousness and indeterminacy", Mind and Language 26 (2): 156-184.

(2011a), Attention and the Indeterminacy of Visual Experience. University of California (Berkeley) $\mathrm{PhD}$ dissertation.

Taylor, J. (2013), "Is attention necessary and sufficient for phenomenal consciousness?", Journal of Consciousness Studies 20 (11-12): 173-194.

Tse, P. U. (2005), "Voluntary attention modulates the brightness of overlapping transparent surfaces", Vision Research 45: 1095-1098.

Tye, M., (2009), Consciousness Revisited: Materialism without Phenomenal Concepts. Cambridge, MA: The MIT Press.

(1995), Ten problems of consciousness: A representational theory of the phenomenal mind. Cambridge, Mass.: MIT Press.

Watzl, S., (2019), "Can Representationalism explain how attention affects Appearances?”, en A. Pautz \& D. Stoljar, eds., Blockheads! Essays on Ned Block's Philosophy of Mind and Consciousness. MIT Press, pp. 581-607.

Wu, W. (2014), Attention. Oxford: Routledge.

(2011), "Attention as Selection for Action", en C. Mole, D. Smithies \& W. Wu, eds., Attention: Philosophical and Psychological Essays. Oxford: Oxford University Press, pp. 247-273, pp. 97-116. 